\title{
Validation of VHTRC calculation benchmark of critical experiment using the MCB code
}

\author{
Przemysław Stanisz ${ }^{1, a}$, Mateusz Malicki ${ }^{1}$ and Mariusz Kopeć ${ }^{1}$ \\ ${ }^{1}$ AGH University of Science and Technology, al. A. Mickiewicza 30, 30-059 Kraków, Poland
}

\begin{abstract}
The calculation benchmark problem Very High Temperature Reactor Critical (VHTR) a pin-in-block type core critical assembly has been investigated with the Monte Carlo Burnup (MCB) code in order to validate the latest version of Nuclear Data Library based on ENDF format. Executed benchmark has been made on the basis of VHTR benchmark available from the International Handbook of Evaluated Reactor Physics Benchmark Experiments. This benchmark is useful for verifying the discrepancies in $\mathrm{k}_{\text {eff }}$ values between various libraries and experimental values. This allows to improve accuracy of the neutron transport calculations that may help in designing the high performance commercial VHTRs. Almost all safety parameters depend on the accuracy of neutron transport calculation results that, in turn depend on the accuracy of nuclear data libraries. Thus, evaluation of the libraries applicability to VHTR modelling is one of the important subjects. We compared the numerical experiment results with experimental measurements using two versions of available nuclear data (ENDF-B-VII.1 and JEFF-3.2) prepared for required temperatures. Calculations have been performed with the MCB code which allows to obtain very precise representation of complex VHTR geometry, including the double heterogeneity of a fuel element. In this paper, together with impact of nuclear data, we discuss also the impact of different lattice modelling inside the fuel pins. The discrepancies of $\mathrm{k}_{\mathrm{eff}}$ have been successfully observed and show good agreement with each other and with the experimental data within the $1 \sigma$ range of the experimental uncertainty. Because some propagated discrepancies observed, we proposed appropriate corrections in experimental constants which can improve the reactivity coefficient dependency. Obtained results confirm the accuracy of the new Nuclear Data Libraries.
\end{abstract}

\section{Introduction}

The objective of the high-temperature gas-cooled reactor benchmark VHTR [1] is to determine the temperature coefficient of reactivity by measuring the temperature dependence of reactivity for the HTGR type reactors. The temperature in large commercial core can change largely between a cold shut-down state and a hot normal operation state. Thereby large change in temperature will impose significant change in reactivity. Proper estimation of the temperature reactivity coefficient is very important as it influences the designed shut-down margin and has significant consequences in economics. Moreover, presented benchmark considers the case with low-enriched $\mathrm{UO}_{2}$ that fits in with IAEA strategy to use as a fuel lowenriched uranium (LEU) rather than high-enriched uranium (HEU).

\section{Description of experiment}

The VHTRC (Very High Temperature Reactor Critical Assembly) assembly is precisely described in „International Handbook of Reactor Physics Experiments Benchmark" [2] distributed by the NEA data Bank. Originally the experiment was located at the JAEA Tokai Research and Development Centre in Japan [3]. The core was designed as thermal, graphite moderated assembly. Each graphite rod is filled with approximately 20000 randomly distributed fuel capsules of the BISOtype The capsule consists of the centrally positioned fuel sphere ( $2 \%$ and $4 \%$ enriched $\mathrm{UO}_{2}$ kernels, respectively) covered by two layers of pyrolytic carbon. Scheme of experimental system is shown on Figure 1. The core prism itself is made of two $2.4 \mathrm{~m}$ height hexagonal assemblies folded in $2.4 \mathrm{~m}$ long core. One assembly is always in fixed position and the second one is movable. Each assembly consists of smaller hexagonal blocks, except the side blocks of trapezoidal shape.

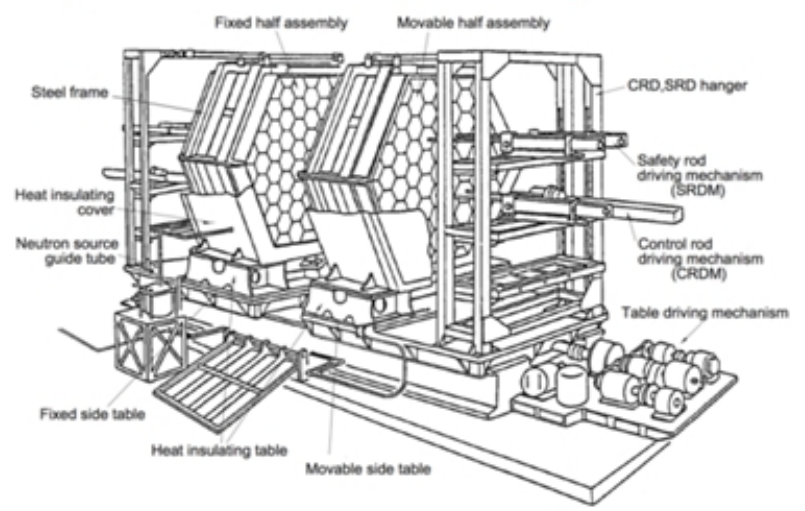

Figure 1. Overhead view of VHTRC

\footnotetext{
${ }^{\mathrm{a}}$ Corresponding author: pstanisz@agh.edu.pl
} 
The original experiment was designed in three different load patterns: HP, HC-1, HC-2. However, present work is focused only on the HP case shown in Figure 2. Core was made mostly with fuel rods called B-4 and one B-2. Difference between them is mainly weight enrichment, respectively $4 \%$ and $2 \%$, but there are also small differences in size. Proposed benchmark based on experiment considers comparison of criticality in 5 temperature points. First criticality measurement was performed in $25.5^{\circ} \mathrm{C}$. In the course of experiments the core was evenly wormed up by electrical heaters until almost $200^{\circ} \mathrm{C}$. Further reactivity values were measured by pulsed neutron method for specific temperatures: $71.2,100.9,150.5$ and $199.6^{\circ} \mathrm{C}$.

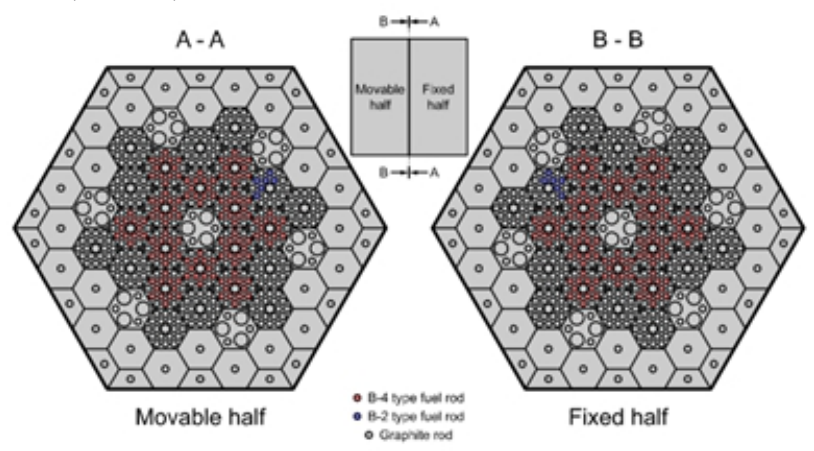

Figure 2. Core Loading Pattern of HP Core

\section{Description of the calculation model}

In order to simulate the benchmark conditions, a numerical representation of VHTR experiment was prepared for the MCB (ver. 5) Monte Carlo code used for calculations[4][5]. Two recent sets of nuclear data: American ENDF-B-VII.1 and European JEFF-3.2 cross section libraries were used. They represent the best available knowledge on the neutron interactions with matter and nuclide properties. It guaranteed the most precise results produced by the MCB code. Performed calculations consider three cases of successive problems that could have impact on obtained results: type of placement of the BISO fuel; temperature cross section applications and recalculation of variables used in presented benchmark.

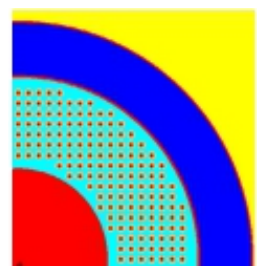

Figure 3. Horizontal sections quarter of or the fuel block with $4 \%$ enrichment, simple cubic.

MCB uses the same geometrical model as the wellknown MCNP code. In the current form direct random placement of fuel kernels is impossible for large number of geometrical elements, however we show in the paper [6] that for models with high packing ratio (as in the considered problem) using random distribution of places could be easy and satisfactory changed using the URAN option. Because of that, for our investigation, two approaches have been introduced in order to understand analysed results:

- Cubic lattice without BISO particle touching the rod surface (simple cubic) - presented in Figure 3,

- Cubic lattice with additional 3D stochastic universe option enabled (cubic URAN) - described by Brown [7].

In case of the second mentioned problem we were faced with temperature interpolation. Typical cross sections are provided for standard temperatures: 300,400 , $500,600,700,800,900,1000,1200$ and $1800 \mathrm{~K}$. On the other hand, the experimental values were determined for very precise temperatures: $298.65,344.35$, $374.05,423.65$ and $472.75 \mathrm{~K}$. Due to this fact we had to do some preparation. Generally there are few main approaches widely used for this:

- direct processing of nuclear data from evaluated nuclear data in the ENDF format;

- manipulation of cross sections library with Doppler broadening of the resolved resonances data and interpolate unresolved resonance probability tables to the new temperatures using higher and lower temperatures;

- calculation for the given library temperatures and interpolation between obtained results;

- mixing two materials of higher and lower standard temperatures and averaging the obtained results (linear or square root interpolation).

Only the first method assumes direct processing of data from the Evaluated Nuclear Data Files and converts them into suitable tables. The other three methods are based on using the ACE data files by MCNP and MCB. For the presented calculation we use the most convenient $2^{\text {nd }}$ method for neutron interaction using the makxsf code delivered with MCNP6. Special treatment with $1^{\text {st }}$ method was carried out only for cross section of the thermal neutrons scattering. In general, thermal cross sections are important in highly thermalized systems, where neutron scattering cross section values are additionally affected by the scattering from waves that are able to interfere with each other in a solid, liquid, or gas. Similarly may contribute the elastic and inelastic scattering of neutrons in materials. Generally additional effects could occur as neutrons give energy to material excitation or gain energy from it. These effects change reaction rates and modify the energy and angle distribution of scattered neutrons. Application of thermal scattering law is represented by two variables $\alpha$ - the momentum transfer and $\beta$ - the energy transfer and is described by function $S(\alpha, \beta)$. This function is prepared within NJOY code [8]. The authors used the LEAPR module, based on General Atomics physics models, to obtain new ENDF files for thermal libraries. Once the data files were complete, they were used into ACE format to recompute the thermal scattering files for used temperatures.

\section{Results}

Application of broadening method performed by the makxsf program and use of the LEAPR module for thermal libraries in order to receive the $S(\alpha, \beta)$ thermal scattering cross section fulfil usefulness into benchmark problem, which results in good agreement concerning 
the temperature interpolation. Results obtained by the authors with generated cross sections - called AGH results - are compared with that of data sets available in the ACE format (NEA sets). All results are plotted in Fig. 4. Interpolated trend curve obtained from the JEFF-3.2 cross sections well overlap with curve from new calculations or produced points. Results are in good agreement. We assume that using the same methodology successful results will be obtained for the ENDF-B-VII.1 cross sections. In the same plot results from the benchmark are presented. Some discrepancy with the experiment is observed at higher temperatures resulting into discrepancies of thermal coefficient of reactivity. As a first approximation, we investigate this problem with simple parametric study.

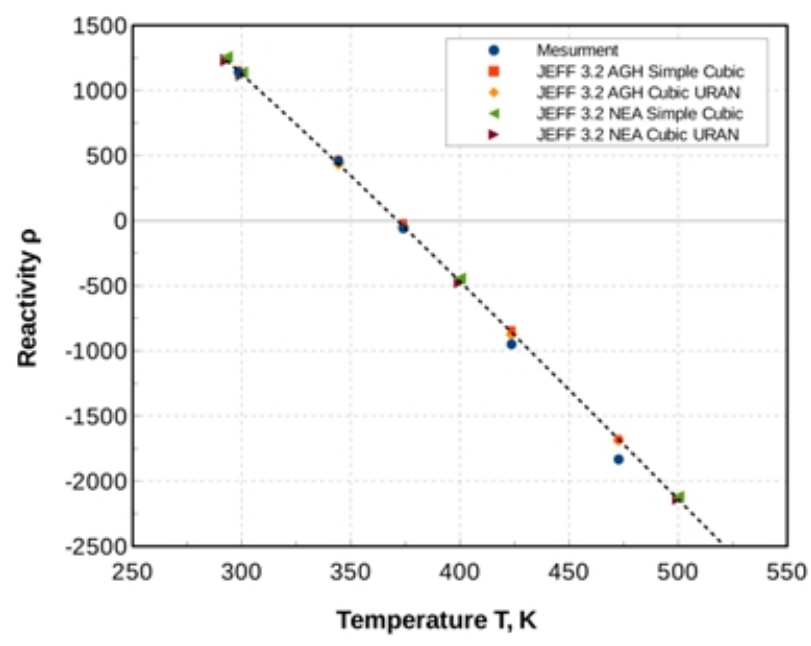

Figure 4. Reactivity change due to assembly temperature rise

In the following step, several parameters have been taken into account in order to see their impact on the thermal coefficient. These are successively: second set of cross section (ENDF-B-VII.1), random placement of the fuel particle through launching the URAN option in the simulation and the impact of distances between BISO particles. In the last approach we increase and decrease the distance by $2 \%$ with the same packing factor. Packing factor of the fuel kernels in considered geometry is very high. However, it is good to see how this parameter will change the results. All results are presented in Figure 5 where temperature dependent differences are compared as a function of reactivity ( $\mathrm{pcm}$ - range) differences between benchmark results and our calculations.

Experimental uncertainty in the benchmark problem is around $300 \mathrm{pcm}$ and our calculation has $6 \mathrm{pcm}$. The $=$ discrepancy between two the farthest cases presented in Figure 5 has roughly $200 \mathrm{pcm}$. Thereby, all results show good agreement with the benchmark data. Numerical results present, on average, a $26 \mathrm{pcm}$ decrease in URAN option compared to the Simple Cubic geometry. Differences between JEFF and ENDF nuclear data library, are not higher than $150 \mathrm{pcm}$. Interestingly, change (in the case of JEFF tables) of local pitch of fuel kernel by $\pm 2 \%$ results in bias of $100 \mathrm{pcm}$. This difference shows that geometry parameters are very viable for different variation of lattice even for high packing factor. The disparity of these results has the same order of magnitude as the differences between JEFF and ENDF data. Therefore, it is almost impossible to indicate which cross section set leads to better agreement with the benchmark problem. Similar problem was studied in our previous work [6] and we found its importance for burnup simulation. Decisively, none of used parametric improvements change the impact on the temperature coefficient. All trend lines have the same inclination. We propose a change of some parameter in order to improve thermal reactivity coefficient, comparing to original paper [4]. Authors of this paper noticed that a few constants (as beta effective or correction values), which are used for recalculation of the reactivity measurement, have been taken from calculations performed with the diffusion code CITATION [9]. To be consistent with only one methodology, in presented analysis, it will be more accurate to use only the Monte Carlo method to obtain all constants. We decided to simulate the experiment by using more suitable routine SOURCE available in MCNP/MCB. Next, Sjöstrand corrected method with spatial effects correction [10] was chosen to calculate area ratio obtained from MCB and recalculate measurement with appropriate correction factor. In original paper [4] $\beta_{\text {eff }}$ was calculated by the CITATION code only for the lowest temperature and was used for all temperatures. It was decided in this work to replace $\beta_{\text {eff }}$ by the values obtained for particular temperatures by the Monte Carlo calculated ones.

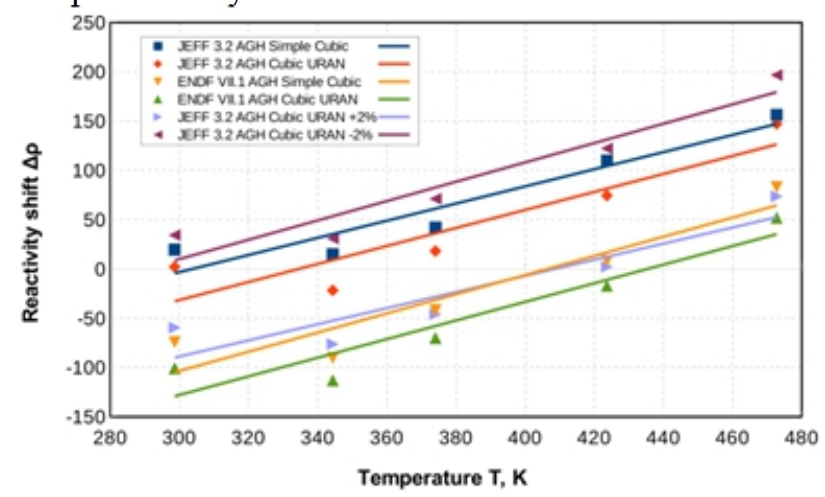

Figure 5. Difference of reactivity in the VHTRC model with various approaches (relative to the benchmark results)

In the original paper [4] areas obtained from $\mathrm{BF}_{4}$ detectors is reported for four temperatures. The Sjöstrand method is strongly affected by spatial effects and corrections of the spatial effects should be done for each detector position. In our calculations we used correction factor (Eq. 1) obtained by the same Monte Carlo method, assuming relation between the expected value of measured reactivity in given detector position and the simulated global reactivity.

$$
f_{\text {cor }}=\frac{\rho_{\text {global,MCNP }}}{\rho_{\text {local,MCNP }}}=\frac{\rho_{\text {global,MCNP }}}{A_{\mathrm{p}, \mathrm{MCNP}} / A_{\mathrm{d}, \mathrm{MCNP}}}
$$

Assumption of the same relation in the experiment provides the corrected value of reactivity that is given by Eq. (2):

$$
\rho_{\text {cor }}=\mathrm{f}_{\text {cor }} \rho_{\text {measur }}
$$


The effective delayed neutron fractions $\beta_{\text {eff }}$ in VHTRC model is calculated by the MCB5 code using prompt transport method (Eqn. 3). This method needs two calculations: first, the normal run (considers prompt and delayed neutrons), and second, only with prompt neutrons:

$$
\beta_{\text {eff }} \simeq 1-\frac{\chi_{p} v_{p}}{\chi v} \simeq 1-\frac{k_{p}}{k}
$$

Differently than in original paper [4], we recalculated $\beta_{\text {eff }}$ for each temperature point. The results of the measurement after applying different methods of correction are listed in Table 1.

Table 1. Benchmark and our values of $\beta_{\text {eff }}$

\begin{tabular}{|c|c|c|}
\hline Temp. [K] & Original $\boldsymbol{\beta}_{\text {eff }}$ & MCB $\boldsymbol{\beta}_{\text {eff }}$ \\
\hline 298.65 & 0.007287 & $0.00710(7)$ \\
\hline 344.35 & 0.007287 & $0.00709(7)$ \\
\hline 374.05 & 0.007287 & $0.00711(7)$ \\
\hline 423.65 & 0.007287 & $0.00712(7)$ \\
\hline 472.75 & 0.007287 & $0.00703(7)$ \\
\hline
\end{tabular}

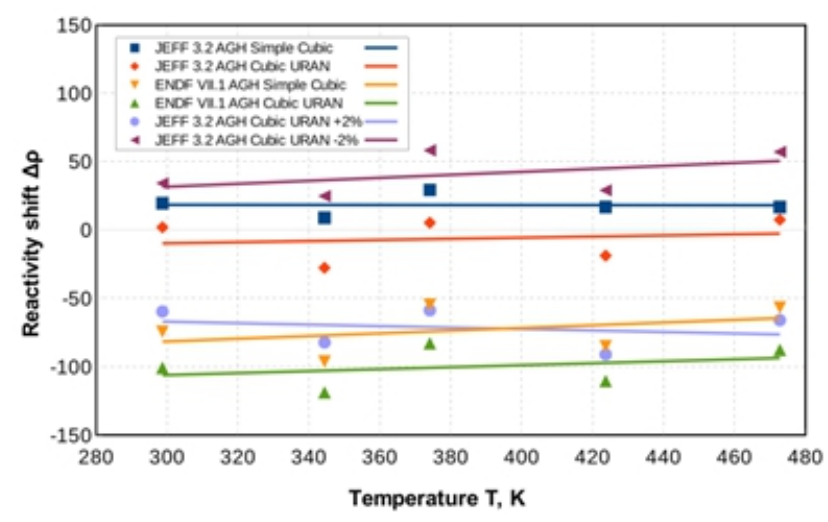

Figure 6. Difference of reactivity in the VHTRC model with various approaches (relative to the updated benchmark results)

\section{Conclusions}

In order to understand temperature effect on reactivity in thermal reactors, extensive benchmarking tests were undertaken to probe the quality of the new data library. As shown here, these studies have considered results of calculations based on the Monte Carlo method compared to results of the critical benchmark measurements. In general, in our calculations we used the most recent available nuclear data in all cases where the results stay within standard uncertainty. However a constant proportional difference in reactivity was observed through increasing the temperature. The performed parametric studies give explanation behaviour on reactivity. It show that in the investigated VHTR model the impact from geometry parameters, regarding fuel arrangement, introduce the same order of uncertainty as the use of different cross section libraries. However, this parametric studies did not improve the temperature reactivity coefficient dependency. It turns out that after recalculation of computable constant values used in the benchmark, we were able to show improvement in simulated experiment results eliminating temperature dependency. Our results suggest taking into account examination of the used computing parameter used in the benchmark measurement and recalculation final reactivity values.

\section{Acknowledgments}

This work was supported by the funds of the Polish Ministry of Science and Higher Education. This research was supported in part by PL-Grid Infrastructure.

\section{References}

1. NEA, "Temperature effect on reactivity in VHTRC-1 core," VHTRC-GCR-EXP-001, CRITCOEF, NEA/NSC/DOC(2006)2, 2006.

2. NEA, International Reactor Physics Experiment Evaluation (IRPhE) Project, https://www.oecdnea.org/science/irphe/irphe-handbook/handbook.html

3. T. Yamane, H. Yasuda, F. Akino, Y. Kaneko J. Nucl. Sci. Technol. 27, 2 (1990)

4. J. Cetnar, User Manual for MCB professional

5. J. Cetnar, M. Kopeć, M. Oettingen, Assessment of Pu and MA utilization in deep burn Prismatic HTR by Monte Carlo Method - MCB - Project PUMA; http://www.kej.agh.edu.pl/publikacje/puma_kej1.pdf

6. G. Kępisty, P. Stanisz, J. Cetnar, Ann. Nucl. Energy. 92 (2016)

7. F.B. Brown, Stochastic Geometry and HTGR Modeling with MCNP5, Conference Paper. The Monte Carlo Method: Versatility Unbounded In A Dynamic Computing World

8. E. MacFarlane, NJOY99-code system for producing pointwise and multigroup neutron and photon crosssections from $E N D F / B$ data, Los Alamos Nat. Laboratory, Los Alamos, NM, Rep. RSIC PSR-480, 2000.

9. T.B. Fowler, D.R. Vondy, G.W. Cunningham, Nuclear Reactor Core Analysis Code CITATION ORNL-TM-2496, Revision 2 (July 1971)

10. P. Gajda, J. Janczyszyn, W. Pohorecki, Nukleonika 58, 2 (2013) 